\title{
A novel AGC scheme in a wideband receiver
}

\author{
Shuigen Huang ${ }^{1,2 a)}$, Min Lin ${ }^{1}$, Zongkun Zhou ${ }^{1,2}$, and Xiaoyun Li ${ }^{1,2}$ \\ ${ }^{1}$ Shanghai Institute of Microsystem and Information Technology, Chinese Academy \\ of Sciences, Shanghai 200050, China \\ ${ }^{2}$ University of Chinese Academy of Sciences, Beijing 100049, China
}

a)huangsg@mail.sim.ac.cn

\begin{abstract}
A new automatic gain control (AGC) scheme in a wideband wireless receiver is presented in this paper. The proposed AGC scheme consists a receiver architecture with tuning the gain of RF amplifier, mixer and IF amplifier synchronously, and a novel exponential function current generator to achieve dB-linear gain control characteristic. Fabricated on a 0.18 um BiCMOS process, the proposed receiver provides a conversion gain dynamic range of $72 \mathrm{~dB}$ with the gain ripple of $5 \mathrm{~dB}$ that range from 0.8 to $2.7 \mathrm{GHz}$. At maximum gain operation, the noise figure of $9.9 \mathrm{~dB}$ and $-25 \mathrm{dBm}$ of third-order input intercept point are measured at $2.7 \mathrm{GHz}$. The chip draws $27 \mathrm{~mW}$ from a $1.8 \mathrm{~V}$ power supply and occupies an area of $2.8 \mathrm{~mm}^{2}$.
\end{abstract}

Keywords: automatic gain control (AGC), receiver, dB-linear, wideband Classification: Integrated circuits

\section{References}

[1] K. Chuang, et al:: "A $90 \mathrm{~nm}$ CMOS broadband multi-mode mixed-signal demodulator for $60 \mathrm{GHz}$ radios," IEEE Trans. Microw. Theory Techn. 58 (2010) 4060 (DOI: 10.1109/TMTT.2010.2084585).

[2] S. Y. Kang, et al:: "A precise decibel-linear programmable gain amplifier using a constant current-density function," IEEE Trans. Microw. Theory Techn. 60 (2012) 2843 (DOI: 10.1109/TMTT.2012.2203928).

[3] A. D. Gore, et al.: "AGC and DCOC algorithms for sliding IF no-coherent ULP wireless receiver,” 13th IEEE CCNC (2016) (DOI: 10.1109/CCNC.2016. 7444879).

[4] G. Jin, et al.: "A stable and two-step settling digital controlled AGC loop for GNSS receiver,” IEICE Electron. Express 11 (2014) 20140738 (DOI: 10.1587/ elex.11.20140738).

[5] S. Chatterjee and H. Rahaman: "Development of AGC and channel equalization algorithm for multi-channel RF system in FPGA," 6th IEEE International Conference on Computers and Devices for Communication (2015) (DOI: 10.1109/CODEC.2015.7893174).

[6] H. Elwan, et al.: "A differential-ramp based $65 \mathrm{~dB}$-linear VGA technique in 65 nm CMOS,” IEEE J. Solid-State Circuits 44 (2009) 2503 (DOI: 10.1109/ JSSC.2009.2021446).

[7] M. Dongi and M. Jalali: “A wideband CMOS VGA with dB-linear gain based on active feedback and negative capacitance," ICEE (2017) (DOI: 10.1109/ IranianCEE.2017.7985090). 
[8] S. Ray and M. M. Hella: "A $10 \mathrm{~Gb} / \mathrm{s}$ inductorless AGC amplifier with $40 \mathrm{~dB}$ linear variable gain control in 0.13 um CMOS," IEEE J. Solid-State Circuits $\mathbf{5 1}$ (2016) 440 (DOI: 10.1109/JSSC.2015.2496782).

[9] D. Bhatt, et al.: "A self-biased mixer in 0.18 um CMOS for an ultra-wideband receiver," IEEE Trans. Microw. Theory Techn. 65 (2017) 1294 (DOI: 10.1109/ TMTT.2016.2640949).

[10] J. Choi, et al:: "Wide dynamic-range CMOS RMS power detector," IEEE Trans. Microw. Theory Techn. 64 (2016) 868 (DOI: 10.1109/TMTT.2016. 2519030).

[11] J. Wang, et al.: "A wide band CMOS radio frequency RMS power detector with 42-dB dynamic range," IEEE International Symposium on Circuits and Systems (ICSAS) (2015) 1678 (DOI: 10.1109/ISCAS.2015.7168974).

\section{Introduction}

The automatic gain control (AGC) is a key component in a wireless receiver. It adjusts the gain of the blocks in the signal path according to the input signal amplitude to avoid clipping distortion experienced by the baseband analogy-todigital converters (ADC) [1]. As the demand for speed and bandwidth of communication system is increasing, the power consumption and data-rate of receivers become a bottleneck for the development of modern receiver. Traditional AGC scheme in receivers is implemented in programmable gain amplifier (PGA) [2], with applying power detector, $\mathrm{ADC}$ and digital processing in physical (PHY) layer to control the gain of PGAs $[3,4,5]$. However, PGAs have some drawbacks like requiring relatively large area overhead due to lots of switches as well as limited gain accuracy due to discrete and finite gain steps. But dB-linear gain control characteristic, which means that the gain in $\mathrm{dB}$ linearly follows the control voltage is a vital requisite to obtain a large dynamic range (DR) along with an independent settling time with respect to input signal power in AGC circuit [6].

In this paper, a new AGC scheme adopting a novel current regulator circuit is presented. Large DR and accurate gain control are both achieved in the scheme. In addition, the architecture of an $R-2 R$ attenuator network with multi-stage variable gain amplifiers (VGA) $[7,8]$ are performed in the design to achieve broadband response that frequencies range from 0.8 to $2.7 \mathrm{GHz}$ [9]. The proposed AGC scheme is applied in RF VGA, Mixer and IF VGA, which means tuning the signal at RF frequency and IF frequency synchronously and optimizing the system signalto-noise ratio (SNR) in an effective method.

The block diagram of the receiver is shown in Fig. 1. The remainder of this paper is organized as follows: In section 2, the AGC scheme in circuit design and analysis are described. Then in section 3, measurement results are presented and discussed. Finally, the results of this work are concluded in section 4. 


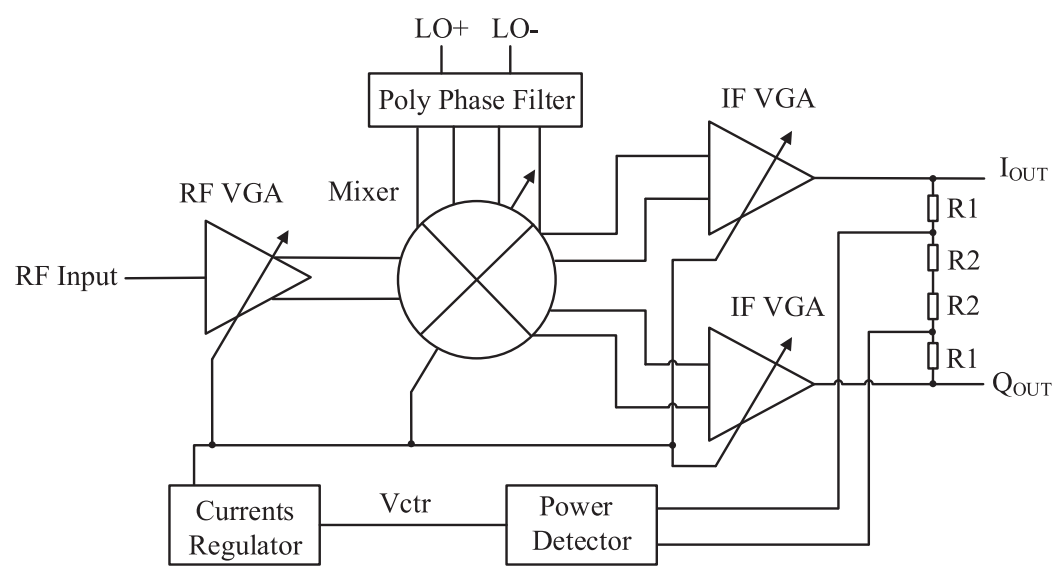

Fig. 1. AGC structure in a wireless receiver

\section{AGC scheme in circuit design}

\subsection{Variable gain amplifier architecture}

In order to achieve $\mathrm{dB}$-linear gain control over a broadband frequency range, a wideband VGA architecture with an $R-2 R$ attenuator network combining a 5-stage VGA is proposed, whose gain is controlled by variable tail currents of the VGA. The architecture is applied in RF VGA, mixer and IF VGA synchronously.

The RF VGA architecture comprises a 5-stage parallel differential amplifier with one of the input being AC-grounded shown in Fig. 2(a), and a 5-stage $R-2 R$

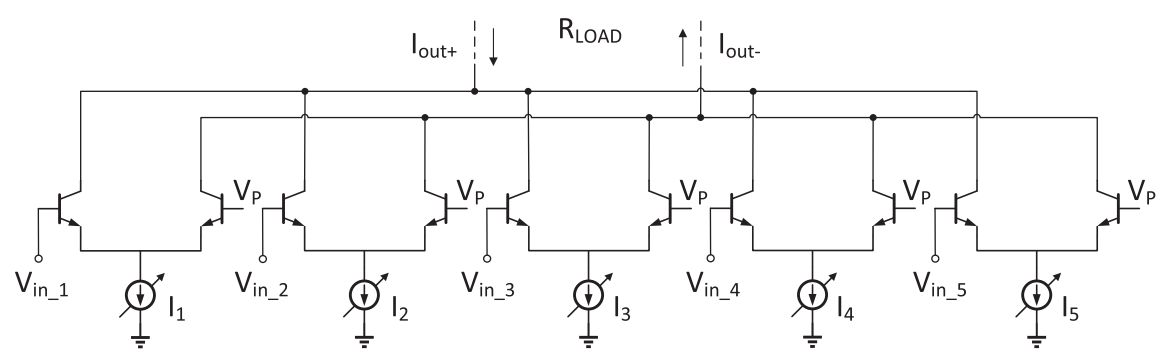

(a)

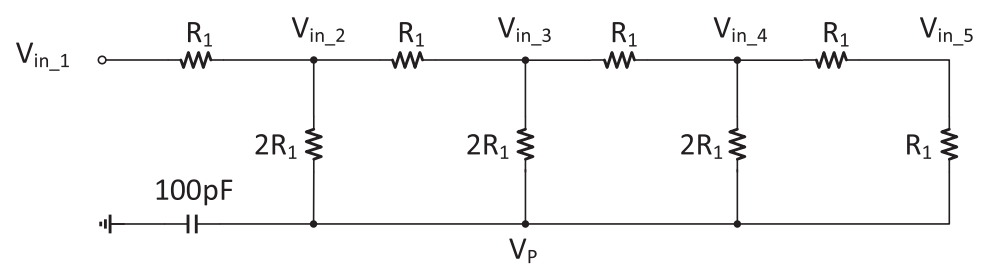

(b)

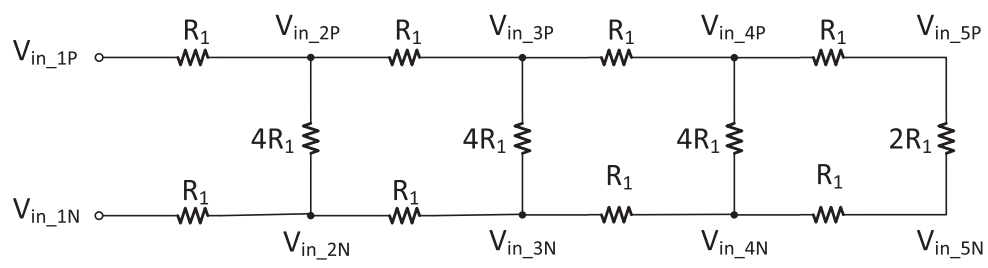

(c) 
attenuator network for single input shown in Fig. 2(b). The input $R-2 R$ attenuator network for differential input is shown in Fig. 2(c), which is applied in the cascade mixer and IF VGA. In Fig. 2(b) and Fig. 2(c), $R_{1}$ is set to $50 \Omega$. Thus, an equivalent resistor of $50 \Omega$ for single input and $100 \Omega$ for differential input is paralleled in each stage amplifier, which is independent of frequency and effective for wideband input matching. In the simulation, the input return loss of lower than $-16 \mathrm{~dB}$ is achieved over the frequency range of 0.8 to $2.7 \mathrm{GHz}$. With the attenuation of $R-2 R$ attenuator network, the input signal for the n-stage amplifier is given by

$$
v_{i n \_n}=\frac{1}{2^{n-1}} \cdot v_{i n \_1} \quad(\mathrm{n}=1 \sim 5)
$$

Assuming the tail current and input signal voltage of the n-stage amplifier are $I_{\mathrm{n}}$ and $v_{\text {in_n } n}$, the output current of the VGA can be expressed as

$$
i_{\text {out }}=\sum_{n=1}^{5} \frac{v_{\text {in_n } n}}{V_{T}} \cdot I_{n}=\left(I_{1}+\frac{I_{2}}{2}+\frac{I_{3}}{4}+\frac{I_{4}}{8}+\frac{I_{5}}{16}\right) \cdot \frac{1}{V_{T}} \cdot v_{\text {in_ } 1}
$$

Where $V_{T}$ is the thermal voltage and can be expressed as

$$
V_{T}=\frac{k T}{q} \approx 26 \mathrm{mV} \quad \text { at } 300^{\circ} \mathrm{K}
$$

Thus, the gain of the RF VGA, related to the tail currents, can be expressed as equation (4), where $R_{L O A D}$ is the load resistor.

$$
G=\frac{v_{\text {out }}}{v_{\text {in }}}=\frac{R_{L O A D}}{V_{T}} \cdot\left(I_{1}+\frac{I_{2}}{2}+\frac{I_{3}}{4}+\frac{I_{4}}{8}+\frac{I_{5}}{16}\right)
$$

\subsection{Current source regulator}

A novel 5-stage current source regulator is proposed to provide tail currents for VGAs as shown in Fig. 3, which is the key component for dB-linear gain tuning. The tail currents are controlled by a DC voltage $\mathrm{V}_{\text {ctr }}$. In this circuit, $\mathrm{I}_{\text {ctrl }}$ is equal to $\mathrm{V}_{\text {ctr }} / \mathrm{R}_{\mathrm{ctr} 1}$, and the sum of current $\mathrm{I}_{\mathrm{ctr} 1}$ and $\mathrm{I}_{\mathrm{ctr} 2}$ is $130 \mathrm{uA}$. The collector current $\mathrm{I}_{\mathrm{C}}$ is set to $26 \mathrm{uA}$, and $\mathrm{R}_{\text {ctr } 1}$ is set to $10 \mathrm{k} \Omega$. Assuming $\mathrm{V}_{\mathrm{n}}$ represent the $\mathrm{DC}$ voltage of point $\mathrm{n}(\mathrm{n}=1 \sim 5)$, with linear superposition analysis, $\mathrm{V}_{1}, \mathrm{~V}_{2}, \mathrm{~V}_{3}, \mathrm{~V}_{4}$ and $\mathrm{V}_{5}$ are

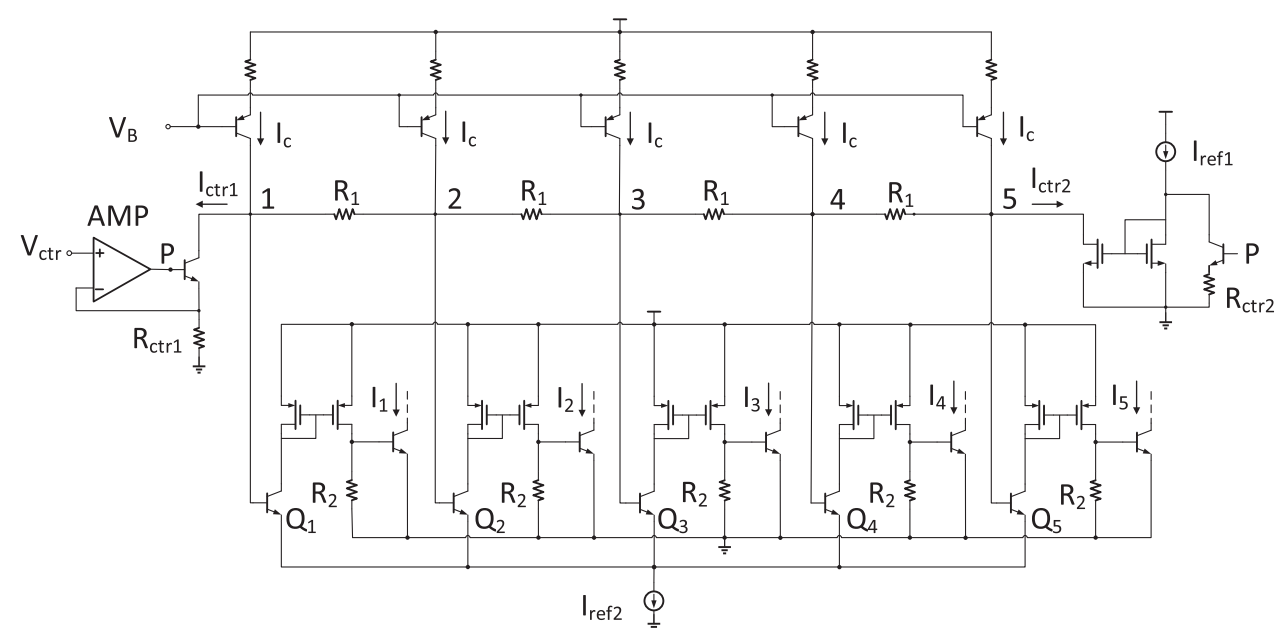

Fig. 3. The architecture of 5-stage current source regulator 
related to the variation voltage drop across resistor $\mathrm{R}_{1}$ tuned by $\mathrm{V}_{\text {ctr }}$. The voltage responses of point 1 to point 5 are shown in Fig. 4(a).

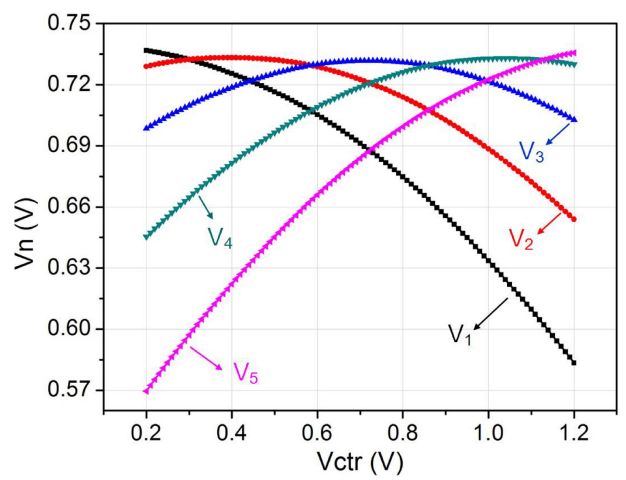

(a)

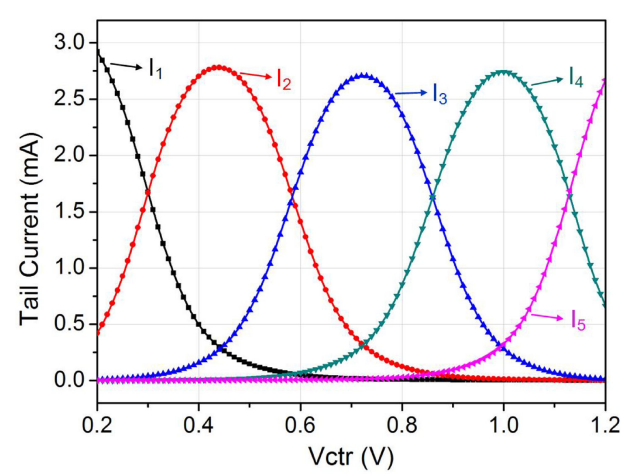

(b)

Fig. 4. (a) The DC voltage of points $1,2,3,4$ and 5 versus $V_{\text {ctr }}$.

(b) The tail currents of RF VGA versus $V_{\text {ctr }}$.

With $\mathrm{V}_{\text {ctr }}$ tuning from 0.2 to $1.2 \mathrm{~V}$, the current $\mathrm{I}_{\text {ctrl }}$ varies from 20 to $120 \mathrm{uA}$, and the maximum voltage among points 1 to 5 varies from point 1 to point 5 in sequence. When the point $\mathrm{n}$ is near to the maximum voltage, the gain of VGA is mainly determined by the $\mathrm{n}$-stage amplifier in Fig. 2, and the tiny voltage variation of point $\mathrm{n}$ tuned by $\mathrm{V}_{\text {ctr }}$ can be expressed as

$$
\Delta V_{n}=\frac{1}{2} \Delta V_{c t r} \cdot \frac{R_{1}}{R_{c t r} 1}
$$

For $\Delta \mathrm{V}_{\mathrm{n}}$ small enough, with reasonable approximation, the variation of the collector current of transistor $\mathrm{Q}_{\mathrm{n}}$ can be expressed as (6). In (6), $I_{c n 0}$ represents the initial collector current of $\mathrm{Q}_{\mathrm{n}}$ when $\Delta \mathrm{V}_{\mathrm{n}}$ is zero.

$$
\Delta I_{c n}=I_{c n 0} \cdot e^{\frac{\Delta V_{n}}{V_{T}}} \approx \frac{I_{c n 0}}{V_{T}} \cdot \Delta V_{n}
$$

Directly tuned by $\Delta \mathrm{I}_{\mathrm{cn}}$, the tail current $\mathrm{I}_{\mathrm{n}}$ can be expressed as equation (7), in which $m$ is constant and determines the slop rate of dB-linear gain tuning.

$$
\Delta I_{n}=I_{n 0} \cdot e^{\frac{\Delta I_{c r} \cdot R_{2}}{V_{T}}}=I_{n 0} \cdot e^{m \cdot \Delta V_{c t r}}
$$

Thus, the voltage gain variation $\Delta \mathrm{G}$ generated from $\Delta \mathrm{V}_{\text {ctr }}$ can be expressed as (8), which is of dB-linear tuning characteristic.

$$
\Delta G \approx \frac{R_{L O A D}}{V_{T}} \cdot \frac{\Delta I_{n}}{2^{n-1}}=K_{n} \cdot e^{m \cdot \Delta V_{c t r}}
$$

The tail currents of RF VGA is shown in Fig. 4(b). As $\mathrm{V}_{\text {ctr }}$ tunes from $0.2 \mathrm{~V}$ to $1.2 \mathrm{~V}$, the tail current $I_{n}$ is tuned in exponentially, and the maximum current transfers from $I_{1}$ to $I_{n}$ in sequence. Accordingly, the gain is determined by the amplifier ranging from the first-stage amplifier to the 5-stage amplifier in sequence. The gain dynamic range (DR) is about $24 \mathrm{~dB}$, as shown in (9).

$$
D R=20 \log \left(2^{4}\right)=24 \mathrm{~dB}
$$




\subsection{AGC closed loop}

The signal path of the receiver including RF VGA, Mixer and IF VGA, the gain of each stage is tuned synchronously with applying the same VGA architecture. The gain of RF VGA is in range $-10 \mathrm{~dB}$ to $14 \mathrm{~dB}$, the gain of mixer in range of $-12 \mathrm{~dB}$ to $12 \mathrm{~dB}$, and the gain of IF VGA in range of $-10 \mathrm{~dB}$ to $14 \mathrm{~dB}$. Thus, the total target gain of the receiver varies from $-32 \mathrm{~dB}$ to $40 \mathrm{~dB}$. In order to realize AGC function, a root mean square (RMS) power detector $[10,11]$ is applied in the design, which detects the amplitude of the IF signal and transforms it to a DC voltage. And then, the DC voltage can be switched to connect with the current source regulator $\mathrm{V}_{\text {ctr }}$ which tunes the gain of VGA blocks dynamically. Thus, the AGC closed loop is formed.

\section{Measurement results}

The microphotograph of the proposed receiver is shown in Fig. 5, which is fabricated in a $0.18 \mathrm{um}$ Bi-CMOS process and occupies an area of $2.8 \mathrm{~mm}^{2}$ including pads. The current consumption of the receiver is $15 \mathrm{~mA}$ with a $1.8 \mathrm{~V}$ power supply.

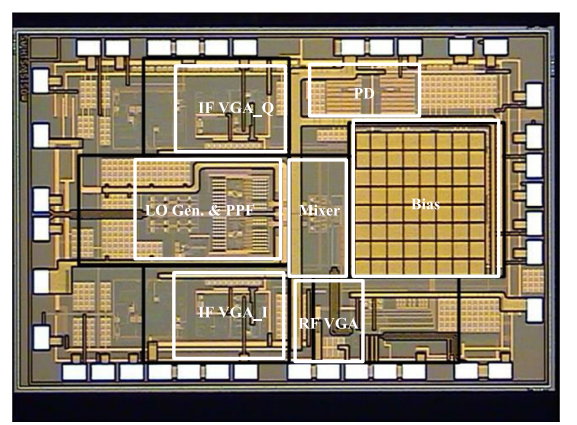

Fig. 5. The die micrograph of the receiver

The measurement results of the input return loss and the conversion gain are shown in Fig. 6(a) and Fig. 6(b) respectively. Over a wideband frequency range of $0.8 \sim 2.7 \mathrm{GHz}$, the $\mathrm{S} 11$ of better than $-7 \mathrm{~dB}$ is measured. Due to the variation of the parasitic effect from package and the characteristic impedance of PCB transmission

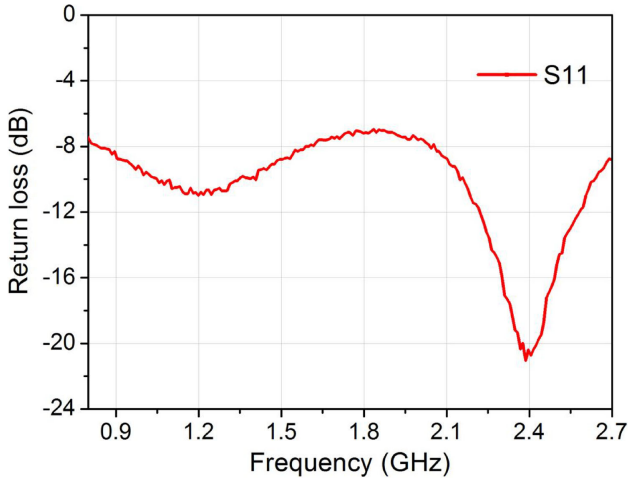

(a)

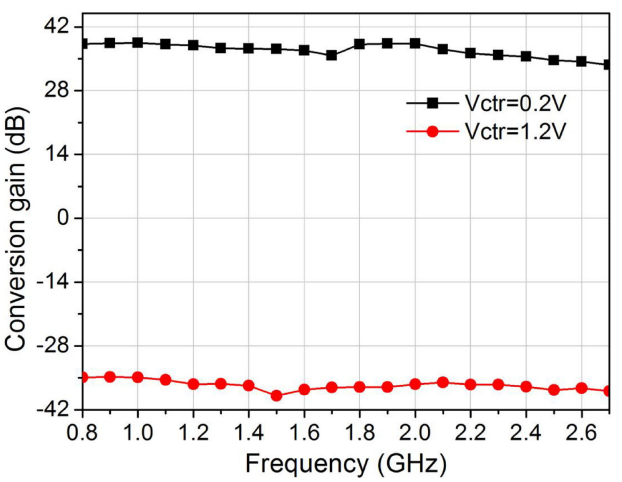

(b) 
line, the input resistance is a little shift from $50 \Omega$ and the measured $\mathrm{S} 11$ is a little worse than the simulated result. The maximum gain variation is $5 \mathrm{~dB}$ among the frequency range 0.8 to $2.7 \mathrm{GHz}$ as shown in Fig. 6(b).

In Fig. 7(a), the conversion gain dynamic range of about $72 \mathrm{~dB}$ is shown. With tuning the gain control voltage $\mathrm{V}_{\mathrm{ctr}}$, the conversion gain at $0.8 \mathrm{GHz}, 1.9 \mathrm{GHz}$, and $2.7 \mathrm{GHz}$ are all plotted. From the figure, the experiment data has a good coherence to the mathematical analysis above. Compared with traditional PGAs, the gain in $\mathrm{dB}$ linearly follows the control voltage with little gain error, which is vital to keep along with the input signal power.

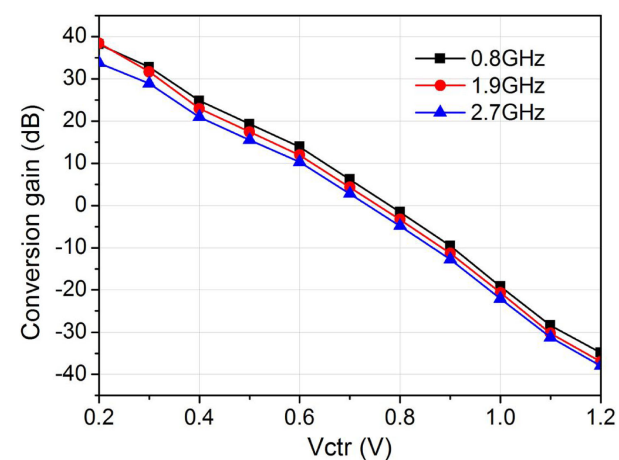

(a)

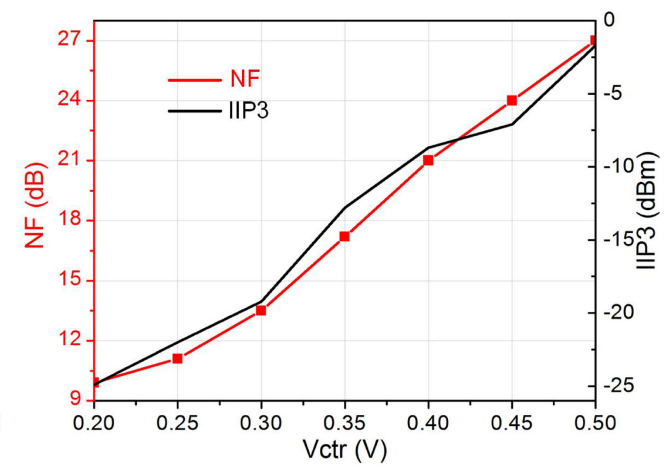

(b)

Fig. 7. (a) Measured conversion gain versus $\mathrm{V}_{\text {ctr }}$. (b) The NF and IIP3 versus $\mathrm{V}_{\mathrm{ctr}}$.

The measured noise figure (NF) and the input third-order intercept point (IIP3) are shown in Fig. 7(b). In the measurement, the LO signal is set to $2.7 \mathrm{GHz}$ with amplitude of $-5 \mathrm{dBm}$, and the two-tone frequencies are set to 2705 and $2706 \mathrm{MHz}$ respectively in the IIP3 measurement. From the figure, the NF of $9.9 \mathrm{~dB}$, and the IIP3 of $-25 \mathrm{dBm}$ are achieved. By tuning the gain control voltage $\mathrm{V}_{\text {ctr }}$, the NF and IIP3 are tuned synchronously, and the signal to noise ratio (SNR) of the demodulated signal is optimized to satisfy the system requirement in an effective method.

\section{Conclusion}

In this letter, a novel AGC scheme in a wideband receiver is introduced. It is target for a wideband application with frequencies range from 0.8 to $2.7 \mathrm{GHz}$. The novel AGC scheme structure consists of an $R-2 R$ attenuator network, an exponential function current generator and a RMS power detector. Only analog circuits are used in circuit design. Larger than $72 \mathrm{~dB}$ conversion gain dynamic range and less than $10 \mathrm{~dB} \mathrm{NF}$ at maximum gain are achieved at the whole band. 